\title{
Antiperiodic Solutions for a Generalized High-Order $(p, q)$-Laplacian Neutral Differential System with Delays in the Critical Case
}

\author{
Yongzhi Liao, ${ }^{1}$ Tianwei Zhang, ${ }^{2}$ and Yongkun $\mathrm{Li}^{3}$ \\ ${ }^{1}$ School of Mathematics and Computer Science, Panzhihua University, Panzhihua, Sichuan 617000, China \\ ${ }^{2}$ City College, Kunming University of Science and Technology, Kunming, Yunnan 650051, China \\ ${ }^{3}$ Department of Mathematics, Yunnan University, Kunming, Yunnan 650091, China
}

Correspondence should be addressed to Yongkun Li; yklie@ynu.edu.cn

Received 23 January 2013; Accepted 13 April 2013

Academic Editor: Anna Capietto

Copyright (C) 2013 Yongzhi Liao et al. This is an open access article distributed under the Creative Commons Attribution License, which permits unrestricted use, distribution, and reproduction in any medium, provided the original work is properly cited.

By applying the method of coincidence degree, some criteria are established for the existence of antiperiodic solutions for a generalized high-order $(p, q)$-Laplacian neutral differential system with delays $\left(\varphi_{p}\left((x(t)-c x(t-\tau))^{(k)}\right)\right)^{(m-k)}=$ $F\left(t, x_{\theta_{0}(t)}, x_{\theta_{1}(t)}^{\prime}, \ldots, x_{\theta_{k}(t)}^{(k)}, y_{\vartheta_{0}(t)}, y_{\vartheta_{1}(t)}^{\prime}, \ldots, y_{\vartheta_{l}(t)}^{(l)}\right),\left(\varphi_{q}\left((y(t)-d y(t-\sigma))^{(l)}\right)\right)^{(n-l)}=G\left(t, y_{\mu_{0}(t)}, y_{\mu_{1}(t)}^{\prime}, \ldots, y_{\mu_{l}(t)}^{(l)}, x_{v_{0}(t)}, x_{v_{1}(t)}^{\prime}, \ldots, x_{v_{k}(t)}^{(k)}\right)$ in the critical case $|c|=|d|=1$. The results of this paper are completely new. Finally, an example is employed to illustrate our results.

\section{Introduction}

During the last twenty years, there have been quite a few results on the existence of periodic solutions for delay differential equations and neutral functional differential equations. We can see [1-7]. For example, the authors of [8-11] investigated the existence of periodic solutions for the following types of neutral functional differential equations:

$$
\begin{gathered}
(x(t)-c x(t-\tau))^{\prime}=g_{1}(t, x(t))+g_{2}(t, x(t-\delta))+e(t), \\
(x(t)-c x(t-\tau))^{\prime \prime}+g(x(t-\delta))=e(t), \\
\left(\varphi_{p}(x(t)-c x(t-\tau))^{\prime}\right)^{\prime}+g(t, x(t-\eta(t)))=e(t), \\
\left(\varphi_{p}\left(x^{\prime}(t)-c x^{\prime}(t-\tau)\right)\right)^{\prime}= \\
f(x(t)) x^{\prime}(t) \\
+\xi(t) g(x(t-\eta(t)))+e(t),
\end{gathered}
$$

respectively. But the condition of constant $|c| \neq 1$ is required. For example, under the assumption $|c| \neq 1$, they obtain that $A: C_{2 \pi}:=\{x: x \in C(\mathbb{R}, \mathbb{R}), x(t+2 \pi) \equiv x(t)\} \rightarrow C_{2 \pi}$,
$(A x)(t)=x(t)-c x(t-\tau)$ has a unique inverse $A^{-1}: C_{2 \pi} \rightarrow$ $C_{2 \pi}$ defined by

$$
\left(A^{-1} f\right)(t)= \begin{cases}\sum_{j \geq 0} c^{j} f(t-j \tau), & |c|<1, \\ -\sum_{j \geq 1} c^{-j} f(t+j \tau), & |c|>1,\end{cases}
$$

and then

$$
\int_{0}^{2 \pi}\left|\left(A^{-1} f\right)(s)\right| \mathrm{d} s \leq \frac{1}{|1-| c||} \int_{0}^{2 \pi}|f(s)| \mathrm{d} s, \quad \forall f \in C_{2 \pi},
$$

which was crucial to obtaining estimation of a priori bounds of periodic solutions in the noncritical case $|c| \neq 1$.

Under the critical case $|c|=1$, the authors of [12-15] studied a first-order neutral differential equation

$$
(x(t)-c x(t-\tau))^{\prime}=g(t, x(t-\delta(t)))+e(t),
$$

a Duffing differential equation of neutral type

$$
(x(t)-c x(t-\tau))^{\prime \prime}=g(t, x(t-\delta(t)))+e(t),
$$


a Rayleigh differential equation of neutral type

$$
\begin{aligned}
(x(t)-c x(t-\tau))^{\prime \prime}= & f(x(t)) x^{\prime}(t) \\
& +g(t, x(t-\delta(t)))+e(t),
\end{aligned}
$$

and a $p$-Laplacian differential equation of neutral type

$$
\begin{aligned}
\left(\varphi_{p}(x(t)-c x(t-\tau))^{\prime}\right)^{\prime}= & f(x(t)) x^{\prime}(t) \\
& +g(t, x(t-\delta(t)))+e(t),
\end{aligned}
$$

respectively.

In the past thirty years, there has been a great deal of work on the problem of the periodic solutions of high-order nonlinear differential equations, especially for the third-order and fourth-order differential equations which have been used to describe nonlinear oscillations [16-20], and fluid mechanical and nonlinear elastic mechanical phenomena [21-27]. In [28], Jin and Lu discussed the existence of periodic solutions of third-order $p$-Laplacian equation with a deviating argument

$$
\begin{gathered}
\left(\varphi_{p}\left(x^{\prime \prime}(t)\right)\right)^{\prime}+f\left(t, x^{\prime}(t), x^{\prime \prime}(t)\right) \\
+g(t, x(t-\eta(t)))=e(t) .
\end{gathered}
$$

Before continuing, by applying Mawhin's continuation theorem of coincidence degree theory, the authors of [29] studied the existence of periodic solutions for a fourth-order $p$-Laplacian equation with a deviating argument

$$
\left(\varphi_{p}\left(x^{\prime \prime}(t)\right)\right)^{\prime \prime}+f\left(x^{\prime \prime}(t)\right)+g(x(t-\eta(t)))=e(t) .
$$

Arising from problems in applied sciences, it is well known that the existence of antiperiodic solutions plays a key role in characterizing the behavior of nonlinear differential equations as a special periodic solution and has been extensively studied by many authors during the past twenty years; see [30-44] and references therein. For example, antiperiodic trigonometric polynomials are important in the study of interpolation problems $[45,46]$, and antiperiodic wavelets are discussed in [47].

However, to the best of our knowledge, due to the neutral term and $p$-Laplace operator term, the existence of antiperiodic solutions for (4)-(7) is very difficult to obtain by applying traditional researching methods. Therefore, to date, there are few papers to investigate the existence of antiperiodic solutions for (4)-(7).
Motivated by above statements, in this paper, we will apply the method of coincidence degree to study the existence of antiperiodic solutions for a generalized high-order $(p, q)$ Laplacian neutral differential system with delays in the critical case

$$
\begin{aligned}
& \left(\varphi_{p}\left((x(t)-c x(t-\tau))^{(k)}\right)\right)^{(m-k)} \\
& \quad=F\left(t, x_{\theta_{0}(t)}, x_{\theta_{1}(t)}^{\prime}, \ldots, x_{\theta_{k}(t)}^{(k)}, y_{\vartheta_{0}(t)}, y_{\vartheta_{1}(t)}^{\prime}, \ldots, y_{\vartheta_{l}(t)}^{(l)}\right), \\
& \left(\varphi_{q}\left((y(t)-d y(t-\sigma))^{(l)}\right)\right)^{(n-l)} \\
& \quad=G\left(t, y_{\mu_{0}(t)}, y_{\mu_{1}(t)}^{\prime}, \ldots, y_{\mu_{l}(t)}^{(l)}, x_{v_{0}(t)}, x_{v_{1}(t)}^{\prime}, \ldots, x_{v_{k}(t)}^{(k)}\right),
\end{aligned}
$$

where $|c|=|d|=1, \varphi_{p}(s)=|s|^{p-2} s, \varphi_{q}(s)=|s|^{q-2} s$, and $s \in \mathbb{R}$, $p, q \geq 2 ; \theta_{i}(t)(0 \leq i \leq k), \vartheta_{i}(t)(0 \leq i \leq l), \mu_{i}(t)(0 \leq i \leq l)$, and $\nu_{i}(t)(0 \leq i \leq k) \in C(\mathbb{R}, \mathbb{R})$ are $\pi$-periodic functions; for any $\rho(t) \in C(\mathbb{R}, \mathbb{R}), x_{\rho(t)}$ is defined by $x_{\rho(t)}=x(t-\rho(t))$; $F, G \in C\left(\mathbb{R}^{k+l+3}, \mathbb{R}\right)$ are $2 \pi$-periodic in their first arguments; $\tau, \sigma$ are constants; $m, n, k$, and $l$ are nonnegative integers, $k<$ $m, l<n$.

Throughout this paper, we will denote by $\mathbb{N}$ the set of nonnegative integers and by $\mathbb{N}_{1}$ the set of odd positive integers.

Let $p=q, k=l, m=n, x=y, c=d, \tau=\sigma$, and $F=G$ in system (10), then system (10) is reformulated as

$$
\begin{aligned}
& \left(\varphi_{p}\left((x(t)-c x(t-\tau))^{(k)}\right)\right)^{(m-k)} \\
& \quad=F\left(t, x_{\theta_{0}(t)}, x_{\theta_{1}(t)}^{\prime}, \ldots, x_{\theta_{k}(t)}^{(k)}, x_{\vartheta_{0}(t)}, x_{\vartheta_{1}(t)}^{\prime}, \ldots, x_{\vartheta_{l}(t)}^{(l)}\right) .
\end{aligned}
$$

Furthermore, one can easily obtain the following.

(a) If $p=q=2, x=y,|c|=|d|=1, k=l=0, m=$ $n=1, \theta_{0}(t)=\delta(t)$

$$
F=F\left(t, x_{\theta_{0}(t)}\right)=g(t, x(t-\delta(t)))+e(t),
$$

then system (10) reduces to (4).

(b) If $p=q=2, x=y,|c|=|d|=1, k=l=0, m=$ $n=2, \theta_{0}(t)=\delta(t)$

$$
F=F\left(t, x_{\theta_{0}(t)}\right)=g(t, x(t-\delta(t)))+e(t),
$$

then system (10) reduces to (5).

(c) If $p=q=2, x=y,|c|=|d|=1, k=l=0, m=$ $n=2, \theta_{0}(t)=\theta_{1}(t) \equiv 0, \vartheta_{0}(t)=\delta(t)$,

$$
\begin{aligned}
F & =F\left(t, x_{\theta_{0}(t)}, x_{\theta_{1}(t)}^{\prime}, x_{\vartheta_{0}(t)}\right) \\
& =f(x(t)) x^{\prime}(t)+g(t, x(t-\delta(t)))+e(t),
\end{aligned}
$$

then system (10) reduces to (6). 
(d) If $p=q, x=y,|c|=|d|=1, k=l=1, m=n=$ $2, \theta_{0}(t)=\theta_{1}(t) \equiv 0, \vartheta_{0}(t)=\delta(t)$,

$$
\begin{aligned}
F & =F\left(t, x_{\theta_{0}(t)}, x_{\theta_{1}(t)}^{\prime}, x_{\vartheta_{0}(t)}\right) \\
& =f(x(t)) x^{\prime}(t)+g(t, x(t-\delta(t)))+e(t),
\end{aligned}
$$

then system (10) reduces to (7).

The main purpose of this paper is to establish sufficient conditions for the existence of $\pi$-antiperiodic solutions to system (10) by using the method of coincidence degree.

The organization of this paper is as follows. In Section 2, we make some preparations. In Section 3, by using the method of coincidence degree, we establish sufficient conditions for the existence of $\pi$-antiperiodic solutions to system (10). An illustrative example is given in Section 4.

\section{Preliminaries}

The following continuation theorem of coincidence degree is crucial in the arguments of our main results.

Lemma 1 (see [48]). Let $\mathbb{X}, \mathbb{Y}$ be two Banach spaces; let $\Omega \subset$ $\mathbb{X}$ be open bounded and symmetric with $0 \in \Omega$. Suppose that $L: D(L) \subset \mathbb{X} \rightarrow \mathbb{Y}$ is a linear Fredholm operator of index zero with $D(L) \cap \bar{\Omega} \neq \emptyset$ and $N: \bar{\Omega} \rightarrow \bigvee$ is L-compact. Further, one also assumes that

(H) $L x-N x \neq \lambda(-L x-N(-x))$, for all $x \in D(L) \cap \partial \Omega, \lambda \in$ $(0,1]$. $\bar{\Omega}$.

Then equation $L x=N x$ has at least one solution on $D(L) \cap$

Definition 2. Let $u(t): \mathbb{R} \rightarrow \mathbb{R}$ be continuous. $u(t)$ is said to be $T / 2$-antiperiodic on $\mathbb{R}$, if

$$
u(t+T)=u(t), \quad u\left(t+\frac{T}{2}\right)=-u(t), \quad \forall t \in \mathbb{R} .
$$

We will adopt the following notations:

$$
\begin{array}{r}
C_{2 \pi}^{k}:=\{u \in C(\mathbb{R}, \mathbb{R}): u \text { is } 2 \pi \text {-periodic }\}, \\
k \in \mathbb{N},|u|_{\infty}=\max _{t \in[0,2 \pi]}|u(t)|,
\end{array}
$$

where $u$ is a $2 \pi$-periodic function.

For the sake of convenience, we introduce the following assumptions.

$\left(H_{1}\right)$ There exist nonnegative constants $\alpha_{1}, \alpha_{2}, \ldots, \alpha_{k+l+2}$, $\beta_{1}, \beta_{2}, \ldots, \beta_{k+l+2}$, such that

$$
\begin{aligned}
& \left|F\left(t, s_{1}, s_{2}, \ldots, s_{k+l+2}\right)-F\left(t, z_{1}, z_{2}, \ldots, z_{k+l+2}\right)\right| \\
& \quad \leq \sum_{i=1}^{k+l+2} \alpha_{i}\left|s_{i}-z_{i}\right|, \\
& \left|G\left(t, s_{1}, s_{2}, \ldots, s_{k+l+2}\right)-G\left(t, z_{1}, z_{2}, \ldots, z_{k+l+2}\right)\right| \\
& \quad \leq \sum_{i=1}^{k+l+2} \beta_{i}\left|s_{i}-z_{i}\right|
\end{aligned}
$$

for any $\left(t, s_{1}, s_{2}, \ldots, s_{k+l+2}\right),\left(t, z_{1}, z_{2}, \ldots, z_{k+l+2}\right) \in$ $\mathbb{R}^{k+l+3}$.

$\left(H_{2}\right)$ For all $\left(t, s_{1}, s_{2}, \ldots, s_{k+l+2}\right) \in \mathbb{R}^{k+l+3}$,

$$
\begin{aligned}
F(t+ & \left.\pi,-s_{1},-s_{2}, \ldots,-s_{k+l+2}\right) \\
& =-F\left(t, s_{1}, s_{2}, \ldots, s_{k+l+2}\right), \\
G(t+ & \left.\pi,-s_{1},-s_{2}, \ldots,-s_{k+l+2}\right) \\
& =-G\left(t, s_{1}, s_{2}, \ldots, s_{k+l+2}\right) .
\end{aligned}
$$

In order to apply Lemma 1 to study the existence of antiperiodic solutions for system (10), we set

$$
\begin{aligned}
\mathbb{X}=\{x= & \left(x_{1}(t), x_{2}(t), y_{1}(t), y_{2}(t)\right)^{T} \in C_{2 \pi}^{k} \times C_{2 \pi}^{m-k-1} \\
& \left.\times C_{2 \pi}^{l} \times C_{2 \pi}^{n-l-1}: x(t+\pi)=-x(t)\right\}, \\
\mathbb{Y}=\{x= & \left(x_{1}(t), x_{2}(t), y_{1}(t), y_{2}(t)\right)^{T} \in C_{2 \pi}^{0} \times C_{2 \pi}^{0} \\
& \left.\times C_{2 \pi}^{0} \times C_{2 \pi}^{0}: x(t+\pi)=-x(t)\right\}
\end{aligned}
$$

are two Banach spaces with the norms

$$
\begin{aligned}
\|x\|_{\mathbb{X}}= & \sum_{i=0}^{k}\left|x_{1}^{(i)}\right|_{\infty}+\sum_{i=0}^{m-k-1}\left|x_{2}^{(i)}\right|_{\infty}+\sum_{i=0}^{l}\left|y_{1}^{(i)}\right|_{\infty} \\
& +\sum_{i=0}^{n-l-1}\left|y_{2}^{(i)}\right|_{\infty} \\
& \|x\|_{\mathbb{Y}}=\sum_{j=1}^{2}\left(\left|x_{j}\right|_{\infty}+\left|y_{j}\right|_{\infty}\right)
\end{aligned}
$$

respectively. Define

$$
\begin{aligned}
\mathbb{D}=\{x= & \left(x_{1}(t), x_{2}(t), y_{1}(t), y_{2}(t)\right)^{T} \in C_{2 \pi}^{k} \times C_{2 \pi}^{m-k} \\
& \left.\times C_{2 \pi}^{l} \times C_{2 \pi}^{n-l}: x(t+\pi)=-x(t)\right\}
\end{aligned}
$$

and two difference operators $A$ and $B$ as follows:

$$
\begin{array}{ll}
A: \mathbb{Y} \longrightarrow \mathbb{Y}, & (A x)(t)=x(t)-c x(t-\tau), \\
B: \mathbb{Y} \longrightarrow \mathbb{Y}, & (B y)(t)=y(t)-d y(t-\sigma) .
\end{array}
$$


Then system (10) reduces to

$$
\begin{gathered}
\left(A x_{1}\right)^{(k)}(t)=\varphi_{p^{\prime}}\left(x_{2}(t)\right), \\
x_{2}^{(m-k)}(t) \\
=F\left(t, x_{1 \theta_{0}(t)}, x_{1 \theta_{1}(t)}^{\prime}, \ldots, x_{1 \theta_{k}(t)}^{(k)}, y_{1 \vartheta_{0}(t)}, y_{1 \vartheta_{1}(t)}^{\prime}, \ldots, y_{\vartheta_{1 l}(t)}^{(l)}\right), \\
\left(B y_{1}\right)^{(l)}(t)=\varphi_{q^{\prime}}\left(y_{2}(t)\right), \\
y_{2}^{(n-l)}(t) \quad \\
=G\left(t, y_{1 \mu_{0}(t)}, y_{1 \mu_{1}(t)}^{\prime}, \ldots, y_{1 \mu_{l}(t)}^{(l)}, x_{1 v_{0}(t)}, x_{1 v_{1}(t)}^{\prime}, \ldots, x_{1 v_{k}(t)}^{(k)}\right),
\end{gathered}
$$

where $1 / p+1 / p^{\prime}=1,1 / q+1 / q^{\prime}=1,1<p^{\prime}, q^{\prime} \leq 2$. Obviously, the existence of antiperiodic solutions to system (10) is equivalent to that of antiperiodic solutions to system (24). Thus, the problem of finding a $\pi$-antiperiodic solution for system (10) reduces to finding one for system (24).

Define a linear operator $L: D(L) \equiv \mathbb{D} \subset \mathbb{X} \rightarrow \mathbb{Y}$ by setting

$$
L x=L\left(\begin{array}{c}
x_{1} \\
x_{2} \\
y_{1} \\
y_{2}
\end{array}\right)=\left(\begin{array}{c}
\left(A x_{1}\right)^{(k)} \\
x_{2}^{(m-k)} \\
\left(B y_{1}\right)^{(l)} \\
y_{2}^{(n-l)}
\end{array}\right), \quad \forall x \in D(L)
$$

and $N: \mathbb{X} \rightarrow \mathbb{Y}$ by setting

$$
\begin{aligned}
N x & =N\left(\begin{array}{l}
x_{1} \\
x_{2} \\
y_{1} \\
y_{2}
\end{array}\right) \\
& =\left(\begin{array}{c}
F\left(t, x_{1 \theta_{0}(t)}, x_{1 \theta_{1}(t)}^{\prime}, \ldots, x_{1 \theta_{k}(t)}^{(k)}, y_{1 \vartheta_{0}(t)}, y_{1 \vartheta_{1}(t)}^{\prime}, \ldots, y_{1 \vartheta_{l}(t)}^{(l)}\right) \\
\varphi_{q^{\prime}}\left(y_{2}(t)\right) \\
G\left(t, y_{1 \mu_{0}(t)}, y_{1 \mu_{1}(t)}^{\prime}, \ldots, y_{1 \mu_{l}(t)}^{(l)}, x_{1 v_{0}(t)}, x_{1 v_{1}(t)}^{\prime}, \ldots, x_{1 v_{k}(t)}^{(k)}\right)
\end{array}\right) .
\end{aligned}
$$

It is easy to see that

$$
\begin{gathered}
\operatorname{Ker} L=\{0\} \\
\operatorname{Im} L=\left\{x \in \mathbb{Y}: \int_{0}^{2 \pi}\left(\begin{array}{l}
x_{1}(s) \\
x_{2}(s) \\
y_{1}(s) \\
y_{2}(s)
\end{array}\right) \mathrm{d} s=\left(\begin{array}{l}
0 \\
0 \\
0 \\
0
\end{array}\right)\right\} \equiv \mathbb{Y} .
\end{gathered}
$$

Thus, $\operatorname{dim} \operatorname{Ker} L=0=\operatorname{codim} \operatorname{Im} L$, and $L$ is a linear Fredholm operator of index zero.

Define the continuous projector $P: \mathbb{X} \rightarrow \operatorname{Ker} L$ and the averaging projector $Q: \mathbb{Y} \rightarrow \mathbb{Y}$ by

$$
P\left(\begin{array}{l}
x_{1} \\
x_{2} \\
y_{1} \\
y_{2}
\end{array}\right)=Q\left(\begin{array}{l}
x_{1} \\
x_{2} \\
y_{1} \\
y_{2}
\end{array}\right)=\frac{1}{2 \pi} \int_{0}^{2 \pi}\left(\begin{array}{l}
x_{1}(s) \\
x_{2}(s) \\
y_{1}(s) \\
y_{2}(s)
\end{array}\right) \mathrm{d} s \equiv\left(\begin{array}{l}
0 \\
0 \\
0 \\
0
\end{array}\right) .
$$

Hence $\operatorname{Im} P=\operatorname{Ker} L$ and $\operatorname{Ker} Q=\operatorname{Im} L$. Denoting by $L_{P}^{-1}$ : $\operatorname{Im} L \rightarrow D(L) \cap \operatorname{Ker} P$ the inverse of $\left.L\right|_{D(L) \cap K e r} P$, we have

$$
\left(L_{P}^{-1}\left(\begin{array}{c}
x_{1} \\
x_{2} \\
y_{1} \\
y_{2}
\end{array}\right)\right)(t)=\left(\begin{array}{c}
\left(A^{-1} K x_{1}\right)(t) \\
\left(K x_{2}\right)(t) \\
\left(B^{-1} K y_{1}\right)(t) \\
\left(K y_{2}\right)(t)
\end{array}\right)
$$

where

$$
\begin{aligned}
& \left(K x_{1}\right)(t)=\sum_{i=0}^{k-1} \frac{1}{i !}\left(A h_{1}\right)^{(i)}(0) t^{i} \\
& +\frac{1}{(k-1) !} \int_{0}^{t}(t-s)^{k-1} x_{1}(s) \mathrm{d} s, \\
& \left(K x_{2}\right)(t)=\sum_{i=0}^{m-k-1} \frac{1}{i !} h_{2}^{(i)}(0) t^{i} \\
& +\frac{1}{(m-k-1) !} \int_{0}^{t}(t-s)^{m-k-1} x_{2}(s) \mathrm{d} s, \\
& \left(K y_{1}\right)(t)=\sum_{i=0}^{l-1} \frac{1}{i !}\left(B h_{3}\right)^{(i)}(0) t^{i} \\
& +\frac{1}{(l-1) !} \int_{0}^{t}(t-s)^{l-1} y_{1}(s) \mathrm{d} s, \\
& \left(K y_{2}\right)(t)=\sum_{i=0}^{n-l-1} \frac{1}{i !} h_{4}^{(i)}(0) t^{i} \\
& +\frac{1}{(n-l-1) !} \int_{0}^{t}(t-s)^{n-l-1} y_{2}(s) \mathrm{d} s,
\end{aligned}
$$

in which $\left(A h_{1}\right)^{(i)}(0)(i=0,1, \ldots, k-1)$ are decided by $E_{1} Z_{1}=$ $B_{1}$, where

$$
E_{1}=\left(\begin{array}{cccccc}
2 & 0 & 0 & \cdots & 0 & 0 \\
c_{1} & 2 & 0 & \cdots & 0 & 0 \\
c_{2} & c_{1} & 2 & \cdots & 0 & 0 \\
\vdots & \vdots & \vdots & & \vdots & \vdots \\
c_{k-2} & c_{k-3} & c_{k-4} & \cdots & 2 & 0 \\
c_{k-1} & c_{k-2} & c_{k-3} & \cdots & c_{1} & 2
\end{array}\right)_{k \times k},
$$

$$
Z_{1}=\left(\begin{array}{c}
\left(A h_{1}\right)^{(k-1)}(0) \\
\left(A h_{1}\right)^{(k-2)}(0) \\
\left(A h_{1}\right)^{(k-3)}(0) \\
\vdots \\
\left(A h_{1}\right)^{\prime}(0) \\
\left(A h_{1}\right)(0)
\end{array}\right)_{k \times 1},
$$


$B_{1}=\left(b_{11}, b_{12}, \ldots, b_{1 k}\right)^{T}, \quad b_{1 i}=-(1 / i !) \int_{0}^{2 \pi}(\pi-s)^{i} x_{1}(s) \mathrm{d} s, c_{j}=$ $(\pi)^{j} / j !$, and $j=1,2, \ldots, k-1 ; h_{2}^{(i)}(0)(i=0,1, \ldots, m-k-1)$ are decided by $E_{2} Z_{2}=B_{2}$, where

$$
\begin{gathered}
E_{2}=\left(\begin{array}{ccccc}
2 & 0 & \cdots & 0 & 0 \\
c_{1} & 2 & \cdots & 0 & 0 \\
c_{2} & c_{1} & \cdots & 0 & 0 \\
\vdots & \vdots & & \vdots & \vdots \\
c_{m-k-2} & c_{m-k-3} & \cdots & 2 & 0 \\
c_{m-k-1} & c_{m-k-2} & \cdots & c_{1} & 2
\end{array}\right)_{(m-k) \times(m-k)} \\
Z_{2}=\left(\begin{array}{c}
h_{2}^{(m-k-1)}(0) \\
h_{2}^{(m-k-2)}(0) \\
h_{2}^{(m-k-3)}(0) \\
\vdots \\
h_{2}^{\prime}(0) \\
h_{2}(0)
\end{array}\right) \\
(m-k) \times 1
\end{gathered}
$$

$B_{2}=\left(b_{21}, b_{22}, \ldots, b_{2(m-k)}\right)^{T}, b_{2 i}=-(1 / i !) \int_{0}^{2 \pi}(\pi-$ $s)^{i} x_{2}(s) \mathrm{d} s, c_{j}=(\pi)^{j} / j !$, and $j=1,2, \ldots, m-k-$ $1 ;\left(B h_{3}\right)^{(i)}(0)(i=0,1, \ldots, l-1)$ are decided by $E_{3} Z_{3}=B_{3}$, where

$$
\begin{gathered}
E_{3}=\left(\begin{array}{cccccc}
2 & 0 & 0 & \cdots & 0 & 0 \\
c_{1} & 2 & 0 & \cdots & 0 & 0 \\
c_{2} & c_{1} & 2 & \cdots & 0 & 0 \\
\vdots & \vdots & \vdots & & \vdots & \vdots \\
c_{l-2} & c_{l-3} & c_{l-4} & \cdots & 2 & 0 \\
c_{l-1} & c_{l-2} & c_{l-3} & \cdots & c_{1} & 2
\end{array}\right)_{l \times l} \\
Z_{3}=\left(\begin{array}{c}
\left(B h_{3}\right)^{(l-1)}(0) \\
\left(B h_{3}\right)^{(l-2)}(0) \\
\left(B h_{3}\right)^{(l-3)}(0) \\
\vdots \\
\left(B h_{3}\right)^{\prime}(0) \\
\left(B h_{3}\right)^{(0)}
\end{array}\right)_{l \times 1},
\end{gathered}
$$

$B_{3}=\left(b_{31}, b_{32}, \ldots, b_{3 l}\right)^{T}, b_{3 i}=-(1 / i !) \int_{0}^{2 \pi}(\pi-s)^{i} y_{1}(s) \mathrm{d} s, c_{j}=$ $(\pi)^{j} / j !$, and $j=1,2, \ldots, l-1 ; h_{4}^{(i)}(0)(i=0,1, \ldots, n-l-1)$ are decided by $E_{4} Z_{4}=B_{4}$, where

$$
E_{4}=\left(\begin{array}{ccccc}
2 & 0 & \cdots & 0 & 0 \\
c_{1} & 2 & \cdots & 0 & 0 \\
c_{2} & c_{1} & \cdots & 0 & 0 \\
\vdots & \vdots & & \vdots & \vdots \\
c_{n-l-2} & c_{n-l-3} & \cdots & 2 & 0 \\
c_{n-l-1} & c_{n-l-2} & \cdots & c_{1} & 2
\end{array}\right)_{(n-l) \times(n-l)}
$$

$$
Z_{4}=\left(\begin{array}{c}
h_{4}^{(n-l-1)}(0) \\
h_{4}^{(n-l-2)}(0) \\
h_{4}^{(n-l-3)}(0) \\
\vdots \\
h_{4}^{\prime}(0) \\
h_{4}(0)
\end{array}\right)_{(n-l) \times 1},
$$

$B_{4}=\left(b_{41}, b_{42}, \ldots, b_{4(n-l)}\right)^{T}, b_{4 i}=-(1 / i !) \int_{0}^{2 \pi}(\pi-$ $s)^{i} y_{2}(s) \mathrm{d} s, c_{j}=(\pi)^{j} / j !$, and $j=1,2, \ldots, n-l-1$.

Clearly, $Q N$ and $L_{P}^{-1}(I-Q) N$ are continuous. Using the Arzela-Ascoli theorem, it is not difficult to show that $\mathrm{QN}(\bar{\Omega}), L_{P}^{-1}(I-Q) N(\bar{\Omega})$ are relatively compact for any open bounded set $\Omega \subset \mathbb{X}$. Therefore, $N$ is $L$-compact on $\bar{\Omega}$ for any open bounded set $\Omega \subset \mathbb{X}$.

Lemma 3 (see [12]). Let $|\tau|=(b / a) \pi$, where $a$ and $b$ are coprime positive integers. Then

(1) if $c=-1, b$ is odd and $a$ is even, then

$\omega_{1}:=\inf _{k \in \mathbb{N}_{1}}\left|1-c e^{-k i \tau}\right|=\inf _{k \in \mathbb{N}_{1}}[2(1+\cos k \tau)]^{1 / 2}>0$

(2) if $c=1$ and $b$ is odd, then

$\omega_{2}:=\inf _{k \in \mathbb{N}_{1}}\left|1-c e^{-k i \tau}\right|=\inf _{k \in \mathbb{N}_{1}}[2(1-\cos k \tau)]^{1 / 2}>0$

(3) if $c=1$ and $a=b=1$, then

$\omega_{3}:=\inf _{k \in \mathbb{N}_{1}}\left|1-c e^{-k i \tau}\right|=\inf _{k \in \mathbb{N}_{1}}[2(1-\cos k \tau)]^{1 / 2}=2>0$.

Lemma 4 (see [13]). Suppose $A: \mathbb{Y} \rightarrow \mathbb{Y},(A x)(t)=x(t)-$ $c x(t-\tau)$ for all $t \in[0,2 \pi]$. Then the following propositions are true.

(1) If $c=-1,|\tau|=(b / a) \pi$, where $a$ and $b$ are coprime positive integers with $b$ odd and $a$ even, then $A$ has a unique inverse $A^{-1}: \mathbb{Y} \rightarrow \mathbb{Y}$ satisfying $\left\|A^{-1}\right\| \leq$ $\left(1 / \omega_{1}\right)$.

(2) If $c=1,|\tau|=(b / a) \pi$, where $a$ and $b$ are coprime positive integers with $b$ odd, then $A$ has a unique inverse $A^{-1}: \mathbb{Y} \rightarrow \mathbb{Y}$ satisfying $\left\|A^{-1}\right\| \leq\left(1 / \omega_{2}\right)$.

(3) If $c=1,|\tau|=\pi$, then $A$ has a unique inverse $A^{-1}$ : $\mathbb{Y} \rightarrow \mathbb{Y}$ satisfying $\left\|A^{-1}\right\| \leq\left(1 / \omega_{3}\right)$.

Lemma 5 (see $[12])$. Let $|\sigma|=(\nu / \mu) \pi$, where $\mu$ and $\nu$ are coprime positive integers. Then

(1) if $d=-1, v$ is odd and $\mu$ is even, then

$\omega_{1}:=\inf _{k \in \mathbb{N}_{1}}\left|1-d e^{-k i \tau}\right|=\inf _{k \in \mathbb{N}_{1}}[2(1+\cos k \tau)]^{1 / 2}>0 ;$ 
(2) if $d=1$ and $v$ is odd, then

$\omega_{2}:=\inf _{k \in \mathbb{N}_{1}}\left|1-d e^{-k i \tau}\right|=\inf _{k \in \mathbb{N}_{1}}[2(1-\cos k \tau)]^{1 / 2}>0 ;$

(3) if $d=1$ and $\mu=\nu=1$, then

$\omega_{3}:=\inf _{k \in \mathbb{N}_{1}}\left|1-d e^{-k i \tau}\right|=\inf _{k \in \mathbb{N}_{1}}[2(1-\cos k \tau)]^{1 / 2}=2>0$.

Lemma 6 (see [13]). Suppose $B: \mathbb{Y} \rightarrow \mathbb{Y},(B x)(t)=x(t)-$ $d x(t-\sigma)$ for all $t \in[0,2 \pi]$. Then the following propositions are true.

(1) If $d=-1,|\sigma|=(\nu / \mu) \pi$, where $\mu$ and $\nu$ are coprime positive integers with $\nu$ odd and $\mu$ even, then $B$ has a unique inverse $B^{-1}: \mathbb{Y} \rightarrow \mathbb{Y}$ satisfying $\left\|B^{-1}\right\| \leq$ $\left(1 / \omega_{1}\right)$.

(2) If $d=1,|\sigma|=(\nu / \mu) \pi$, where $\mu$ and $\nu$ are coprime positive integers with $v$ odd, then $B$ has a unique inverse $B^{-1}: \mathbb{Y} \rightarrow \mathbb{Y}$ satisfying $\left\|B^{-1}\right\| \leq\left(1 / \omega_{2}\right)$.

(3) If $d=1,|\sigma|=\pi$, then $B$ has a unique inverse $B^{-1}$ : $\mathbb{Y} \rightarrow \mathbb{Y}$ satisfying $\left\|B^{-1}\right\| \leq\left(1 / \omega_{3}\right)$.

\section{Main Result}

In this section, we will study the existence of $\pi$-antiperiodic solutions for system (10) in the critical case $|c|=|d|=1$.

Theorem 7. Assume that $\left(H_{1}\right)-\left(H_{2}\right)$ hold. Suppose further that $c=-1,|\tau|=(b / a) \pi$, where $a$ and $b$ are coprime positive integers with $b$ odd and $a$ even, then system (10) has at least one $\pi$-antiperiodic solution, if one of the following conditions holds.

(1) $d=-1,|\sigma|=(\nu / \mu) \pi$, where $\mu$ and $\nu$ are coprime positive integers with $\nu$ odd and $\mu$ even, and $W_{1}+W_{2}<$ 1 , where

$W_{1}=\max \left\{\sum_{i=1}^{k+1} \frac{\pi^{m-i+1}}{\omega_{1}} \alpha_{i}, \sum_{i=1}^{k+1} \frac{\pi^{n-l+k-i+1}}{\omega_{1}} \beta_{l+i+1}\right\}$,

$W_{2}=\max \left\{\sum_{i=1}^{l+1} \frac{\pi^{m-k+l-i+1}}{\omega_{1}} \alpha_{k+i+1}, \sum_{i=1}^{l+1} \frac{\pi^{n-i+1}}{\omega_{1}} \beta_{i}\right\}$.

(2) $d=1,|\sigma|=(\nu / \mu) \pi$, where $\mu$ and $\nu$ are coprime positive integers with $v$ odd, and $W_{1}+W_{3}<1$, where

$$
W_{3}=\max \left\{\sum_{i=1}^{l+1} \frac{\pi^{m-k+l-i+1}}{\omega_{2}} \alpha_{k+i+1}, \sum_{i=1}^{l+1} \frac{\pi^{n-i+1}}{\omega_{2}} \beta_{i}\right\} .
$$

(3) $d=1,|\sigma|=\pi$, and $W_{1}+W_{4}<1$, where

$$
W_{4}=\max \left\{\sum_{i=1}^{l+1} \frac{\pi^{m-k+l-i+1}}{\omega_{3}} \alpha_{k+i+1}, \sum_{i=1}^{l+1} \frac{\pi^{n-i+1}}{\omega_{3}} \beta_{i}\right\},
$$

in which $\omega_{1}, \omega_{2}$, and $\omega_{3}$ are constants defined by Lemma 3 or Lemma 5.

Proof. As the proof of other cases works almost exactly as the proof of case (1), we will prove case (1) only. Consider the operator equation

$$
L x-N x=\lambda(-L x-N(-x)), \quad \lambda \in(0,1] .
$$

Then we have

$$
\begin{aligned}
\left(A x_{1}\right)^{(k)}(t) & =\frac{1}{1+\lambda} \varphi_{p^{\prime}}\left(x_{2}(t)\right)-\frac{\lambda}{1+\lambda} \varphi_{p^{\prime}}\left(-x_{2}(t)\right), \\
x_{2}^{(m-k)}(t) & =\frac{1}{1+\lambda} F(t, x, y)-\frac{\lambda}{1+\lambda} F(t,-x,-y), \\
\left(B y_{1}\right)^{(l)}(t) & =\frac{1}{1+\lambda} \varphi_{q^{\prime}}\left(y_{2}(t)\right)-\frac{\lambda}{1+\lambda} \varphi_{q^{\prime}}\left(-y_{2}(t)\right), \\
y_{2}^{(n-l)}(t) & =\frac{1}{1+\lambda} G(t, y, x)-\frac{\lambda}{1+\lambda} G(t,-y,-x),
\end{aligned}
$$

where

$$
\begin{aligned}
& F\left(t, x_{1}, y_{1}\right) \\
& =F\left(t, x_{1 \theta_{0}(t)}, x_{1 \theta_{1}(t)}^{\prime}, \ldots, x_{1 \theta_{k}(t)}^{(k)}, y_{1 \vartheta_{0}(t)}, y_{1 \vartheta_{1}(t)}^{\prime}, \ldots, y_{1 \vartheta_{l}(t)}^{(l)}\right), \\
& G\left(t, y_{1}, x_{1}\right) \\
& =G\left(t, y_{1 \mu_{0}(t)}, y_{1 \mu_{1}(t)}^{\prime}, \ldots, y_{1 \mu_{l}(t)}^{(l)}, x_{1 v_{0}(t)}, x_{1 v_{1}(t)}^{\prime}, \ldots, x_{1 v_{k}(t)}^{(k)}\right), \\
& F\left(t,-x_{1},-y_{1}\right) \\
& =F\left(t,-x_{1 \theta_{0}(t)},-x_{1 \theta_{1}(t)}^{\prime}, \ldots,-x_{1 \theta_{k}(t)}^{(k)},-y_{1 \vartheta_{0}(t)},-y_{1 \vartheta_{1}(t)}^{\prime},\right. \\
& \left.\ldots,-y_{1 \vartheta_{l}(t)}^{(l)}\right) \text {, } \\
& G\left(t,-y_{1},-x_{1}\right) \\
& =G\left(t,-y_{1 \mu_{0}(t)},-y_{1 \mu_{1}(t)}^{\prime}, \ldots,-y_{1 \mu_{l}(t)}^{(l)},-x_{1 v_{0}(t)},-x_{1 v_{1}(t)}^{\prime},\right. \\
& \left.\ldots,-x_{1 v_{k}(t)}^{(k)}\right) \text {. }
\end{aligned}
$$

Suppose that $x(t)=\left(x_{1}(t), x_{2}(t), y_{1}(t), y_{2}(t)\right)^{T} \in \mathbb{D}$ is an arbitrary $\pi$-antiperiodic solution of system (45). Because $x_{1}(t) \in C_{2 \pi}^{k}$ is $T / 2$-antiperiodic, hence, we have

$$
\begin{aligned}
\int_{0}^{2 \pi} x_{1}(s) \mathrm{d} s & =\int_{0}^{\pi} x_{1}(s) \mathrm{d} s+\int_{\pi}^{2 \pi} x_{1}(s) \mathrm{d} s \\
& =\int_{0}^{\pi} x_{1}(s) \mathrm{d} s+\int_{0}^{\pi} x_{1}(s+\pi) \mathrm{d} s=0 .
\end{aligned}
$$


Then there exists a constant $\xi \in[0,2 \pi]$ such that

$$
x_{1}(\xi)=0
$$

Therefore, we have

$$
\begin{aligned}
\left|x_{1}(t)\right| & =\left|x_{1}(\xi)+\int_{\xi}^{t} x_{1}^{\prime}(s) \mathrm{d} s\right| \leq \int_{\xi}^{t}\left|x_{1}^{\prime}(s)\right| \mathrm{d} s \\
\left|x_{1}(t)\right| & =\left|x_{1}(t-2 \pi)\right|=\left|x_{1}(\xi)-\int_{t-2 \pi}^{\xi} x_{1}^{\prime}(s) \mathrm{d} s\right| \\
& \leq \int_{t-2 \pi}^{\xi}\left|x_{1}^{\prime}(s)\right| \mathrm{d} s
\end{aligned}
$$

for all $t \in[\xi, \xi+2 \pi]$. Combining the above two inequalities, we can get

$$
\begin{aligned}
\left|x_{1}\right|_{\infty} & =\max _{t \in[0,2 \pi]}\left|x_{1}(t)\right| \\
& =\max _{t \in[\xi, \xi+2 \pi]}\left|x_{1}(t)\right| \\
& \leq \max _{t \in[\xi, \xi+2 \pi]}\left\{\frac{1}{2}\left(\int_{\xi}^{t}\left|x_{1}^{\prime}(s)\right| \mathrm{d} s+\int_{t-2 \pi}^{\xi}\left|x_{1}^{\prime}(s)\right| \mathrm{d} s\right)\right\} \\
& \leq \frac{1}{2} \int_{0}^{2 \pi}\left|x_{1}^{\prime}(s)\right| \mathrm{d} s .
\end{aligned}
$$

Similar to (50), one can easily get

$$
\begin{gathered}
\left|x_{1}^{\prime}\right|_{\infty} \leq \frac{1}{2} \int_{0}^{2 \pi}\left|x_{1}^{\prime \prime}(s)\right| \mathrm{d} s \\
\left|x_{1}^{\prime \prime}\right|_{\infty} \leq \frac{1}{2} \int_{0}^{2 \pi}\left|x_{1}^{\prime \prime \prime}(s)\right| \mathrm{d} s, \ldots,\left|x_{1}^{(k-1)}\right|_{\infty} \leq \frac{1}{2} \int_{0}^{2 \pi}\left|x_{1}^{(k)}(s)\right| \mathrm{d} s
\end{gathered}
$$

which yield

$$
\left|x_{1}^{(i)}\right|_{\infty} \leq \pi^{k-i}\left|x_{1}^{(k)}\right|_{\infty} \quad \text { for } i=0,1, \ldots, k-1 .
$$

By a parallel argument to (47)-(52), we can also obtain

$$
\begin{gathered}
\left|y_{1}^{(i)}\right|_{\infty} \leq \pi^{l-i}\left|y_{1}^{(l)}\right|_{\infty}, \quad \text { for } i=0,1, \ldots, l-1, \\
\left|x_{2}^{(j)}\right|_{\infty} \leq \pi^{m-k-j-1}\left|x_{2}^{(m-k-1)}\right|_{\infty}, \quad \text { for } j=0,1, \ldots, m-k-2, \\
\left|y_{2}^{(j)}\right|_{\infty} \leq \pi^{n-l-j-1}\left|y_{2}^{(n-l-1)}\right|_{\infty}, \quad \text { for } j=0,1, \ldots, n-l-2 .
\end{gathered}
$$

Since $x_{2}(t) \in C_{2 \pi}^{m-k}$ is $\pi$-antiperiodic, similar to (47), there exists a constant $\zeta$ such that $x_{2}^{(m-k-1)}(\zeta)=0$. By a parallel argument to (50), we can obtain from $(45),\left(H_{1}\right),(52)$, and (53) that

$$
\begin{aligned}
& 2\left|x_{2}^{(m-k-1)}\right|_{\infty} \\
& \leq 2\left|x_{2}^{(m-k-1)}(\zeta)\right|+\int_{0}^{2 \pi}\left|x_{2}^{(m-k)}(s)\right| \mathrm{d} s \\
& =\int_{0}^{2 \pi}\left|\frac{1}{1+\lambda} F\left(s, x_{1}, y_{1}\right)-\frac{\lambda}{1+\lambda} F\left(s,-x_{1},-y_{1}\right)\right| \mathrm{d} s \\
& \leq \frac{1}{1+\lambda} \int_{0}^{2 \pi}\left|F\left(s, x_{1}, y_{1}\right)\right| \mathrm{d} s \\
& +\frac{\lambda}{1+\lambda} \int_{0}^{2 \pi}\left|F\left(s,-x_{1},-y_{1}\right)\right| \mathrm{d} s \\
& \leq \frac{1}{1+\lambda} \int_{0}^{2 \pi}\left|F\left(s, x_{1}, y_{1}\right)-F(s, 0,0)\right| \mathrm{d} s \\
& +\frac{\lambda}{1+\lambda} \int_{0}^{2 \pi}\left|F\left(s,-x_{1},-y_{1}\right)-F(s, 0,0)\right| \mathrm{d} s \\
& +\frac{1}{1+\lambda} \int_{0}^{2 \pi}|F(s, 0,0)| \mathrm{d} s+\frac{\lambda}{1+\lambda} \int_{0}^{2 \pi}|F(s, 0,0)| \mathrm{d} s \\
& \leq \frac{1}{1+\lambda} \int_{0}^{2 \pi}\left(\alpha_{1}\left|x_{1}\left(s-\theta_{0}(s)\right)\right|+\alpha_{2}\left|x_{1}^{\prime}\left(s-\theta_{1}(s)\right)\right|+\cdots\right. \\
& +\alpha_{k+1}\left|x_{1}^{(k)}\left(s-\theta_{k}(s)\right)\right| \\
& +\alpha_{k+2}\left|y_{1}\left(s-\vartheta_{0}(s)\right)\right| \\
& +\alpha_{k+3}\left|y_{1}^{\prime}\left(s-\vartheta_{1}(s)\right)\right|+\cdots \\
& \left.+\alpha_{k+l+2}\left|y_{1}^{(l)}\left(s-\vartheta_{l}(s)\right)\right|\right) \mathrm{d} s \\
& +\frac{\lambda}{1+\lambda} \int_{0}^{2 \pi}\left(\alpha_{1}\left|x_{1}\left(s-\theta_{0}(s)\right)\right|+\cdots\right. \\
& +\alpha_{k+1}\left|x_{1}^{(k)}\left(s-\theta_{k}(s)\right)\right| \\
& +\alpha_{k+2}\left|y_{1}\left(s-\vartheta_{0}(s)\right)\right|+\cdots \\
& \left.+\alpha_{k+l+2}\left|y_{1}^{(l)}\left(s-\vartheta_{l}(s)\right)\right|\right) \mathrm{d} s \\
& +\int_{0}^{2 \pi}|F(s, 0,0)| \mathrm{d} s \\
& \leq \int_{0}^{2 \pi}\left(\alpha_{1}\left|x_{1}\left(s-\theta_{0}(s)\right)\right|+\alpha_{2}\left|x_{1}^{\prime}\left(s-\theta_{1}(s)\right)\right|+\cdots\right. \\
& +\alpha_{k+1}\left|x_{1}^{(k)}\left(s-\theta_{k}(s)\right)\right|+\alpha_{k+2}\left|y_{1}\left(s-\vartheta_{0}(s)\right)\right| \\
& +\alpha_{k+3}\left|y_{1}^{\prime}\left(s-\vartheta_{1}(s)\right)\right|+\cdots \\
& \left.+\alpha_{k+l+2}\left|y_{1}^{(l)}\left(s-\vartheta_{l}(s)\right)\right|\right) \mathrm{d} s+\int_{0}^{2 \pi}|F(s, 0,0)| \mathrm{d} s
\end{aligned}
$$




$$
\begin{aligned}
& \leq \int_{0}^{2 \pi}\left(\alpha_{1}\left|x_{1}\right|_{\infty}+\alpha_{2}\left|x_{1}^{\prime}\right|_{\infty}+\cdots+\alpha_{k}\left|x_{1}^{(k-1)}\right|_{\infty}\right. \\
& +\alpha_{k+1}\left|x_{1}^{(k)}\right|_{\infty}+\alpha_{k+2}\left|y_{1}\right|_{\infty}+\alpha_{k+3}\left|y_{1}^{\prime}\right|_{\infty}+\cdots \\
& \left.+\alpha_{k+l+1}\left|y_{1}^{(l-1)}\right|_{\infty}+\alpha_{k+l+2}\left|y_{1}^{(l)}\right|_{\infty}\right) \mathrm{d} s \\
& +\int_{0}^{2 \pi}|F(s, 0,0)| \mathrm{d} s \\
& \leq 2 \pi \alpha_{1}\left|x_{1}\right|_{\infty}+2 \pi \alpha_{2}\left|x_{1}^{\prime}\right|_{\infty}+\cdots+2 \pi \alpha_{k}\left|x_{1}^{(k-1)}\right|_{\infty} \\
& +2 \pi \alpha_{k+1}\left|x_{1}^{(k)}\right|_{\infty}+2 \pi \alpha_{k+2}\left|y_{1}\right|_{\infty}+2 \pi \alpha_{k+3}\left|y_{1}^{\prime}\right|_{\infty}+\cdots \\
& +2 \pi \alpha_{k+l+1}\left|y_{1}^{(l-1)}\right|_{\infty}+2 \pi \alpha_{k+l+2}\left|y_{1}^{(l)}\right|_{\infty} \\
& +2 \pi \max _{s \in[0,2 \pi]}|F(s, 0,0)| \\
& \leq 2 \pi \alpha_{1} \frac{(2 \pi)^{k}}{2^{k}}\left|x_{1}^{(k)}\right|_{\infty}+2 \pi \alpha_{2} \frac{(2 \pi)^{k-1}}{2^{k-1}}\left|x_{1}^{(k)}\right|_{\infty}+\cdots \\
& +2 \pi \alpha_{k} \frac{2 \pi}{2}\left|x_{1}^{(k)}\right|_{\infty}+2 \pi \alpha_{k+1}\left|x_{1}^{(k)}\right|_{\infty} \\
& +2 \pi \alpha_{k+2} \frac{(2 \pi)^{l}}{2^{l}}\left|y_{1}^{(l)}\right|_{\infty}+2 \pi \alpha_{k+3} \frac{(2 \pi)^{l-1}}{2^{l-1}}\left|y_{1}^{(l)}\right|_{\infty} \\
& +\cdots+2 \pi \alpha_{k+l+1} \frac{2 \pi}{2}\left|y_{1}^{(l)}\right|_{\infty}+2 \pi \alpha_{k+l+2}\left|y_{1}^{(l)}\right|_{\infty} \\
& +2 \pi \max _{s \in[0,2 \pi]}|F(s, 0,0)| \\
& =2 \sum_{i=1}^{k+1} \pi^{k-i+2} \alpha_{i}\left|x_{1}^{(k)}\right|_{\infty}+2 \sum_{i=1}^{l+1} \pi^{l-i+2} \alpha_{k+i+1}\left|y_{1}^{(l)}\right|_{\infty} \\
& +2 \pi \max _{s \in[0,2 \pi]}|F(s, 0,0)| .
\end{aligned}
$$

Namely,

$$
\begin{aligned}
\left|x_{2}^{(m-k-1)}\right|_{\infty} \leq & \sum_{i=1}^{k+1} \pi^{k-i+2} \alpha_{i}\left|x_{1}^{(k)}\right|_{\infty}+\sum_{i=1}^{l+1} \pi^{l-i+2} \alpha_{k+i+1}\left|y_{1}^{(l)}\right|_{\infty} \\
& +\pi \max _{s \in[0,2 \pi]}|F(s, 0,0)|
\end{aligned}
$$

Since $y_{2}(t) \in C_{2 \pi}^{n-l}$ is $\pi$-antiperiodic, similar to (50), there exists a constant $\eta$ such that $y_{2}^{(n-l-1)}(\eta)=0$. By a parallel argument to (50), we can obtain from $(45),\left(H_{1}\right),(52)$, and (53) that

$$
\begin{aligned}
& 2\left|y_{2}^{(n-l-1)}\right|_{\infty} \\
& \quad \leq 2\left|y_{2}^{(n-l-1)}(\eta)\right|+\int_{0}^{2 \pi}\left|y_{2}^{(n-l)}(s)\right| \mathrm{d} s \\
& \quad=\int_{0}^{2 \pi}\left|\frac{1}{1+\lambda} G\left(s, y_{1}, x_{1}\right)-\frac{\lambda}{1+\lambda} G\left(s,-y_{1},-x_{1}\right)\right| \mathrm{d} s
\end{aligned}
$$

$\leq \frac{1}{1+\lambda} \int_{0}^{2 \pi}\left|G\left(s, y_{1}, x_{1}\right)\right| \mathrm{d} s$

$+\frac{\lambda}{1+\lambda} \int_{0}^{2 \pi}\left|G\left(s,-y_{1},-x_{1}\right)\right| \mathrm{d} s$

$\leq \frac{1}{1+\lambda} \int_{0}^{2 \pi}\left|G\left(s, y_{1}, x_{1}\right)-G(s, 0,0)\right| \mathrm{d} s$

$+\frac{\lambda}{1+\lambda} \int_{0}^{2 \pi}\left|G\left(s,-y_{1},-x_{1}\right)-G(s, 0,0)\right| \mathrm{d} s$

$+\frac{1}{1+\lambda} \int_{0}^{2 \pi}|G(s, 0,0)| \mathrm{d} s+\frac{\lambda}{1+\lambda} \int_{0}^{2 \pi}|G(s, 0,0)| \mathrm{d} s$

$\leq \frac{1}{1+\lambda} \int_{0}^{2 \pi}\left(\beta_{1}\left|y_{1}\left(s-\mu_{0}(s)\right)\right|\right.$

$+\beta_{2}\left|y_{1}^{\prime}\left(s-\mu_{1}(s)\right)\right|+\cdots$

$+\beta_{l+1}\left|y_{1}^{(l)}\left(s-\mu_{l}(s)\right)\right|$

$+\beta_{l+2}\left|x_{1}\left(s-v_{0}(s)\right)\right|$

$+\beta_{l+3}\left|x_{1}^{\prime}\left(s-v_{1}(s)\right)\right|+\cdots$

$\left.+\beta_{l+k+2}\left|x_{1}^{(k)}\left(s-v_{k}(s)\right)\right|\right) \mathrm{d} s$

$+\frac{\lambda}{1+\lambda} \int_{0}^{2 \pi}\left(\beta_{1}\left|y_{1}\left(s-\mu_{0}(s)\right)\right|\right.$

$$
\begin{aligned}
& +\beta_{2}\left|y_{1}^{\prime}\left(s-\mu_{1}(s)\right)\right|+\cdots \\
& +\beta_{l+1}\left|y_{1}^{(l)}\left(s-\mu_{l}(s)\right)\right| \\
& +\beta_{l+2}\left|x_{1}\left(s-v_{0}(s)\right)\right| \\
& +\beta_{l+3}\left|x_{1}^{\prime}\left(s-v_{1}(s)\right)\right|+\cdots \\
& \left.+\beta_{l+k+2}\left|x_{1}^{(k)}\left(s-v_{k}(s)\right)\right|\right) \mathrm{d} s
\end{aligned}
$$

$$
\begin{aligned}
& +\int_{0}^{2 \pi}|G(s, 0,0)| \mathrm{d} s \\
& \leq \int_{0}^{2 \pi}\left(\beta_{1}\left|y_{1}\left(s-\mu_{0}(s)\right)\right|+\beta_{2}\left|y_{1}^{\prime}\left(s-\mu_{1}(s)\right)\right|\right. \\
& \quad+\cdots+\beta_{l+1}\left|y_{1}^{(l)}\left(s-\mu_{l}(s)\right)\right| \\
& \quad+\beta_{l+2}\left|x_{1}\left(s-v_{0}(s)\right)\right|+\beta_{l+3}\left|x_{1}^{\prime}\left(s-v_{1}(s)\right)\right| \\
& \left.+\cdots+\beta_{l+k+2}\left|x_{1}^{(k)}\left(s-v_{k}(s)\right)\right|\right) \mathrm{d} s \\
& +\int_{0}^{2 \pi}\left(\beta_{1}\left|y_{1}\right|_{\infty}+\beta_{2}\left|y_{1}^{\prime}\right|_{\infty}+\cdots+\beta_{l}\left|y_{1}^{(l-1)}\right|_{\infty}\right. \\
& \quad+\beta_{l+1}\left|y_{1}^{(l)}\right|_{\infty}+\beta_{l+2}\left|x_{1}\right|_{\infty}+\beta_{l+3}\left|x_{1}^{\prime}\right|_{\infty}+\cdots \\
& \left.\quad+\beta_{l+k+1}\left|x_{1}^{(k-1)}\right|_{\infty}+\beta_{l+k+2}\left|x_{1}^{(k)}\right|_{\infty}\right) \mathrm{d} s \\
& +\int_{0}^{2 \pi}|G(s, 0,0)| \mathrm{d} s
\end{aligned}
$$




$$
\begin{aligned}
\leq & 2 \pi \beta_{1}\left|y_{1}\right|_{\infty}+2 \pi \beta_{2}\left|y_{1}^{\prime}\right|_{\infty}+\cdots+2 \pi \beta_{l}\left|y_{1}^{(l-1)}\right|_{\infty} \\
& +2 \pi \beta_{l+1}\left|y_{1}^{(l)}\right|_{\infty}+2 \pi \beta_{l+2}\left|x_{1}\right|_{\infty}+2 \pi \beta_{l+3}\left|x_{1}^{\prime}\right|_{\infty}+\cdots \\
& +2 \pi \beta_{l+k+1}\left|x_{1}^{(k-1)}\right|_{\infty}+2 \pi \beta_{l+k+2}\left|x_{1}^{(k)}\right|_{\infty} \\
& +2 \pi \max _{s \in[0,2 \pi]}|G(s, 0,0)| \\
\leq & 2 \pi \beta_{1} \frac{(2 \pi)^{l}}{2^{l}}\left|y_{1}^{(l)}\right|_{\infty}+2 \pi \beta_{2} \frac{(2 \pi)^{l-1}}{2^{l-1}}\left|y_{1}^{(l)}\right|_{\infty}+\cdots \\
& +2 \pi \beta_{l} \frac{2 \pi}{2}\left|y_{1}^{(l)}\right|_{\infty}+2 \pi \beta_{l+1}\left|y_{1}^{(l)}\right|_{\infty}+\left.\left.2 \pi \beta_{l+2} \frac{(2 \pi)^{k}}{2^{k}}\right|_{1} ^{(k)}\right|_{\infty} \\
& +2 \pi \beta_{l+3} \frac{(2 \pi)^{k-1}}{2^{k-1}}\left|x_{1}^{(k)}\right|_{\infty}+\cdots+2 \pi \beta_{l+k+1} \frac{2 \pi}{2}\left|x_{1}^{(k)}\right|_{\infty} \\
& +2 \pi \beta_{l+k+2}\left|x_{1}^{(k)}\right|_{\infty}+2 \pi \max _{s \in[0,2 \pi]}|G(s, 0,0)| \\
& +2 \pi \max _{s \in[0,2 \pi]}|G(s, 0,0)| . \\
= & 2 \sum_{i=1}^{l+1} \pi^{l-i+2} \beta_{i}\left|y_{1}^{(l)}\right|_{\infty}+2 \sum_{i=1}^{k+1} \pi^{k-i+2} \beta_{l+i+1}\left|x_{1}^{(k)}\right|_{\infty} \\
& +2 \pi x^{2}
\end{aligned}
$$

Namely,

$$
\begin{aligned}
\left|y_{2}^{(n-l-1)}\right|_{\infty} \leq & \sum_{i=1}^{l+1} \pi^{l-i+2} \beta_{i}\left|y_{1}^{(l)}\right|_{\infty}+\sum_{i=1}^{k+1} \pi^{k-i+2} \beta_{l+i+1}\left|x_{1}^{(k)}\right|_{\infty} \\
& +\pi \max _{s \in[0,2 \pi]}|G(s, 0,0)|
\end{aligned}
$$

From (1) of Lemmas 4 and 6, one can obtain

$$
\begin{aligned}
\left|x_{1}^{(k)}(t)\right| & =\left|A^{-1} A x_{1}^{(k)}(t)\right| \leq \frac{1}{\omega_{1}}\left|A x_{1}^{(k)}(t)\right| \\
& =\frac{1}{\omega_{1}}\left|\left(A x_{1}\right)^{(k)}(t)\right| \leq \frac{1}{\omega_{1}} \varphi_{p^{\prime}}\left(\left|x_{2}\right|_{\infty}\right), \\
\left|y_{1}^{(l)}(t)\right| & =\left|B^{-1} B y_{1}^{(l)}(t)\right| \leq \frac{1}{\omega_{1}}\left|B x_{1}^{(l)}(t)\right| \\
& =\frac{1}{\omega_{1}}\left|\left(B y_{1}\right)^{(l)}(t)\right| \leq \frac{1}{\omega_{1}} \varphi_{q^{\prime}}\left(\left|y_{2}\right|_{\infty}\right) .
\end{aligned}
$$

That is,

$$
\left|x_{1}^{(k)}\right|_{\infty} \leq \frac{1}{\omega_{1}} \varphi_{p^{\prime}}\left(\left|x_{2}\right|_{\infty}\right), \quad\left|y_{1}^{(l)}\right|_{\infty} \leq \frac{1}{\omega_{1}} \varphi_{q^{\prime}}\left(\left|y_{2}\right|_{\infty}\right) .
$$

From (54) and (55), we can get

$$
\left|x_{2}\right|_{\infty} \leq \pi^{m-k-1}\left|x_{2}^{(m-k-1)}\right|_{\infty}, \quad\left|y_{2}\right|_{\infty} \leq \pi^{n-l-1}\left|y_{2}^{(n-l-1)}\right|_{\infty} .
$$

With (57)-(62), we have

$$
\begin{aligned}
\left|x_{2}\right|_{\infty} \leq & \sum_{i=1}^{k+1} \frac{\pi^{m-i+1}}{\omega_{1}} \alpha_{i}\left|x_{2}\right|_{\infty}^{p^{\prime-1}} \\
& +\sum_{i=1}^{l+1} \frac{\pi^{m-k+l-i+1}}{\omega_{1}} \alpha_{k+i+1}\left|y_{2}\right|_{\infty}^{q^{\prime}-1}+T_{1}, \\
\left|y_{2}\right|_{\infty} \leq & \sum_{i=1}^{k+1} \frac{\pi^{n-l+k-i+1}}{\omega_{1}} \beta_{l+i+1}\left|x_{2}\right|_{\infty}^{p^{\prime}-1} \\
& +\sum_{i=1}^{l+1} \frac{\pi^{n-i+1}}{\omega_{1}} \beta_{i}\left|y_{2}\right|_{\infty}^{q^{\prime}-1}+T_{2},
\end{aligned}
$$

where

$$
\begin{aligned}
& T_{1}=\pi^{m-k} \max _{s \in[0,2 \pi]}|F(s, 0,0)|, \\
& T_{2}=\pi^{n-l} \max _{s \in[0,2 \pi]}|G(s, 0,0)| .
\end{aligned}
$$

Let $X_{0}=\max \left\{\left|x_{2}\right|_{\infty},\left|y_{2}\right|_{\infty}\right\}$. Then we have from (63) that

$$
X_{0} \leq W_{1} X_{0}^{p^{\prime}-1}+W_{2} X_{0}^{q^{\prime}-1}+\max \left\{T_{1}, T_{2}\right\}
$$

in which

$$
\begin{aligned}
& W_{1}=\max \left\{\sum_{i=1}^{k+1} \frac{\pi^{m-i+1}}{\omega_{1}} \alpha_{i}, \sum_{i=1}^{k+1} \frac{\pi^{n-l+k-i+1}}{\omega_{1}} \beta_{l+i+1}\right\}, \\
& W_{2}=\max \left\{\sum_{i=1}^{l+1} \frac{\pi^{m-k+l-i+1}}{\omega_{1}} \alpha_{k+i+1}, \sum_{i=1}^{l+1} \frac{\pi^{n-i+1}}{\omega_{1}} \beta_{i}\right\} .
\end{aligned}
$$

Since $1<p^{\prime}, q^{\prime} \leq 2,(65)$ implies from (1) that there exists a positive constant $M_{0}$ such that $X_{0} \leq M_{0}$; that is,

$$
\left|x_{2}\right|_{\infty} \leq M_{0}, \quad\left|y_{2}\right|_{\infty} \leq M_{0},
$$

which implies from (61) that there exist positive constants $M_{1}$ and $M_{2}$ such that

$$
\left|x_{1}^{(k)}\right|_{\infty} \leq M_{1}, \quad\left|y_{1}^{(l)}\right|_{\infty} \leq M_{2}
$$

which implies from (57) and (59) that

$$
\begin{aligned}
\left|x_{2}^{(m-k-1)}\right|_{\infty} \leq & \sum_{i=1}^{k+1} \pi^{k-i+2} \alpha_{i} M_{1}+\sum_{i=1}^{l+1} \pi^{l-i+2} \alpha_{k+i+1} M_{2} \\
& +\pi \max _{s \in[0,2 \pi]}|F(s, 0,0)| \equiv M_{3}, \\
\left|y_{2}^{(n-l-1)}\right|_{\infty} \leq & \sum_{i=1}^{l+1} \pi^{l-i+2} \beta_{i} M_{2}+\sum_{i=1}^{k+1} \pi^{k-i+2} \beta_{l+i+1} M_{1} \\
& +\pi \max _{s \in[0,2 \pi]}|G(s, 0,0)| \equiv M_{4} .
\end{aligned}
$$


Substituting (68), (69) into (52), (53), (54), and (55), we get

$$
\begin{aligned}
\left|x_{1}^{(i)}\right|_{\infty} \leq & \frac{T^{k-i}}{2^{k-i}}\left|x_{1}^{(k)}\right|_{\infty} \\
& \leq \frac{T^{k-i}}{2^{k-i}} M_{1} \equiv N_{i} \quad \text { for } i=0,1, \ldots, k-1, \\
\left|y_{1}^{(i)}\right|_{\infty} \leq & \frac{T^{l-i}}{2^{l-i}}\left|y_{1}^{(l)}\right|_{\infty} \\
\leq & \frac{T^{l-i}}{2^{l-i}} M_{2} \equiv L_{i} \quad \text { for } i=0,1, \ldots, l-1, \\
\left|x_{2}^{(j)}\right|_{\infty} \leq & \frac{T^{m-k-j-1}}{2^{m-k-j-1}}\left|x_{2}^{(m-k-1)}\right|_{\infty} \\
\leq & \frac{T^{m-k-j-1}}{2^{m-k-j-1}} M_{3} \equiv H_{j} \quad \text { for } j=0,1, \ldots, m-k-2, \\
\left|y_{2}^{(j)}\right|_{\infty} \leq & \frac{T^{n-l-j-1}}{2^{n-l-j-1}}\left|y_{2}^{(n-l-1)}\right|_{\infty} \\
\leq & \frac{T^{n-l-j-1}}{2^{n-l-j-1}} M_{4} \equiv K_{j} \quad \text { for } j=0,1, \ldots, n-l-2 .
\end{aligned}
$$

Let

$$
\begin{aligned}
M= & \sum_{i=0}^{k-1} N_{i}+\sum_{i=0}^{m-k-2} H_{i}+\sum_{i=0}^{l-1} L_{i}+\sum_{i=0}^{n-l-2} K_{i} \\
& +\sum_{i=1}^{4} M_{i}+1(\text { Clearly, } M \text { is independent of } \lambda) .
\end{aligned}
$$

Take

$$
\Omega=\left\{x \in \mathbb{X}:\|x\|_{\mathbb{X}}<M\right\} .
$$

It is clear that $\Omega$ satisfies all the requirements in Lemma 1 and condition $(H)$ is satisfied. In view of all the discussions above, we conclude from Lemma 1 that system (10) has at least one $\pi$-antiperiodic solution. This completes the proof.

Similar to Theorem 7, we can easily obtain the following results.

Theorem 8. Assume that $\left(H_{1}\right)-\left(H_{2}\right)$ hold. Suppose further that $c=1,|\tau|=(b / a) \pi$, where $a$ and $b$ are coprime positive integers with $b$ odd, then system (10) has at least one $\pi$-antiperiodic solution, if one of the following conditions holds.

(1) $d=-1,|\sigma|=(\nu / \mu) \pi$, where $\mu$ and $\nu$ are coprime positive integers with $\nu$ odd and $\mu$ even, and $W_{5}+W_{2}<$ 1 , where

$$
\begin{aligned}
& W_{5}=\max \left\{\sum_{i=1}^{k+1} \frac{\pi^{m-i+1}}{\omega_{2}} \alpha_{i}, \sum_{i=1}^{k+1} \frac{\pi^{n-l+k-i+1}}{\omega_{2}} \beta_{l+i+1}\right\}, \\
& W_{2}=\max \left\{\sum_{i=1}^{l+1} \frac{\pi^{m-k+l-i+1}}{\omega_{1}} \alpha_{k+i+1}, \sum_{i=1}^{l+1} \frac{\pi^{n-i+1}}{\omega_{1}} \beta_{i}\right\} .
\end{aligned}
$$

(2) $d=1,|\sigma|=(\nu / \mu) \pi$, where $\mu$ and $\nu$ are coprime positive integers with $v$ odd, and $W_{5}+W_{3}<1$, where

$$
W_{3}=\max \left\{\sum_{i=1}^{l+1} \frac{\pi^{m-k+l-i+1}}{\omega_{2}} \alpha_{k+i+1}, \sum_{i=1}^{l+1} \frac{\pi^{n-i+1}}{\omega_{2}} \beta_{i}\right\} .
$$

(3) $d=1,|\sigma|=\pi$, and $W_{5}+W_{4}<1$, where

$$
W_{4}=\max \left\{\sum_{i=1}^{l+1} \frac{\pi^{m-k+l-i+1}}{\omega_{3}} \alpha_{k+i+1}, \sum_{i=1}^{l+1} \frac{\pi^{n-i+1}}{\omega_{3}} \beta_{i}\right\},
$$

in which $\omega_{1}, \omega_{2}$, and $\omega_{3}$ are constants defined by Lemma 3 or Lemma 5.

Theorem 9. Assume that $\left(H_{1}\right)-\left(H_{2}\right)$ hold. Suppose further that $c=1,|\tau|=\pi$, then system (10) has at least one $\pi$-antiperiodic solution, if one of the following conditions holds.

(1) $d=-1,|\sigma|=(\nu / \mu) \pi$, where $\mu$ and $\nu$ are coprime positive integers with $\nu$ odd and $\mu$ even, and $W_{6}+W_{2}<$ 1 , where

$$
\begin{aligned}
& W_{6}=\max \left\{\sum_{i=1}^{k+1} \frac{\pi^{m-i+1}}{\omega_{3}} \alpha_{i}, \sum_{i=1}^{k+1} \frac{\pi^{n-l+k-i+1}}{\omega_{3}} \beta_{l+i+1}\right\}, \\
& W_{2}=\max \left\{\sum_{i=1}^{l+1} \frac{\pi^{m-k+l-i+1}}{\omega_{1}} \alpha_{k+i+1}, \sum_{i=1}^{l+1} \frac{\pi^{n-i+1}}{\omega_{1}} \beta_{i}\right\} .
\end{aligned}
$$

(2) $d=1,|\sigma|=(\nu / \mu) \pi$, where $\mu$ and $\nu$ are coprime positive integers with $\nu$ odd, and $W_{6}+W_{3}<1$, where

$$
W_{3}=\max \left\{\sum_{i=1}^{l+1} \frac{\pi^{m-k+l-i+1}}{\omega_{2}} \alpha_{k+i+1}, \sum_{i=1}^{l+1} \frac{\pi^{n-i+1}}{\omega_{2}} \beta_{i}\right\} \text {. }
$$

(3) $d=1,|\sigma|=\pi$, and $W_{6}+W_{4}<1$, where

$$
W_{4}=\max \left\{\sum_{i=1}^{l+1} \frac{\pi^{m-k+l-i+1}}{\omega_{3}} \alpha_{k+i+1}, \sum_{i=1}^{l+1} \frac{\pi^{n-i+1}}{\omega_{3}} \beta_{i}\right\},
$$

in which $\omega_{1}, \omega_{2}$, and $\omega_{3}$ are constants defined by Lemma 3 or Lemma 5.

\section{An Example}

Example 1. Let $p, q \geq 2$. Then the following third-order $(p, q)$-Laplacian neutral differential system

$$
\begin{aligned}
& \left(\varphi_{p}\left(\left(x(t)+x\left(t-\frac{\pi}{2}\right)\right)^{\prime}\right)\right)^{\prime \prime} \\
& \quad=\sin t+\frac{\sqrt{2}}{\pi^{4}} x(t-1)+\frac{\sqrt{2}}{\pi^{6}} y(t-1)+\frac{\sqrt{2}}{\pi^{6}} y^{\prime}(t-1), \\
& \left(\varphi_{q}\left(\left(y(t)+y\left(t-\frac{\pi}{2}\right)\right)^{\prime \prime}\right)\right)^{\prime} \\
& =\cos t+\frac{\sqrt{2}}{\pi^{6}} y(t-1)+\frac{\sqrt{2}}{\pi^{4}} x(t-1)
\end{aligned}
$$

has at least one $\pi$-antiperiodic solution. 
Proof. By calculation, $\omega_{1}=\sqrt{2}, \alpha_{1}=\sqrt{2} / \pi^{4}, \alpha_{3}=\alpha_{4}=$ $\sqrt{2} / \pi^{6}, \alpha_{2}=\alpha_{5}=0, \beta_{1}=\sqrt{2} / \pi^{6}, \beta_{4}=\sqrt{2} / \pi^{4}$, and $\beta_{2}=$ $\beta_{3}=\beta_{5}=0$. Therefore,

$$
\begin{gathered}
W_{1}=\max \left\{\frac{1}{\pi}, \frac{1}{\pi^{2}}\right\}=\frac{1}{\pi}<\frac{1}{2}, \\
W_{2}=\max \left\{\frac{1}{\pi^{2}}+\frac{1}{\pi^{3}}, \frac{1}{\pi^{3}}\right\}=\frac{1}{\pi^{2}}+\frac{1}{\pi^{3}}<\frac{1}{2} .
\end{gathered}
$$

Hence,

$$
W_{1}+W_{2}<\frac{1}{2}+\frac{1}{2}=1
$$

which implies that case (1) in Theorem 7 holds. It is easy to verify that $\left(H_{1}\right)-\left(H_{2}\right)$ hold and the result follows from Theorem 7. This completes the proof.

\section{Acknowledgment}

This work is supported by the National Natural Sciences Foundation of People's Republic of China under Grant 10971183.

\section{References}

[1] E. Serra, "Periodic solutions for some nonlinear differential equations of neutral type," Nonlinear Analysis. Theory, Methods \& Applications, vol. 17, no. 2, pp. 139-151, 1991.

[2] M. R. Zhang, "Periodic solutions of linear and quasilinear neutral functional-differential equations," Journal of Mathematical Analysis and Applications, vol. 189, no. 2, pp. 378-392, 1995.

[3] S. P. Lu and W. G. Ge, "Periodic solutions for a kind of Liénard equation with a deviating argument," Journal of Mathematical Analysis and Applications, vol. 289, no. 1, pp. 231-243, 2004.

[4] S. P. Lu and W. G. Ge, "Some new results on the existence of periodic solutions to a kind of Rayleigh equation with a deviating argument," Nonlinear Analysis. Theory, Methods \& Applications, vol. 56, no. 4, pp. 501-514, 2004.

[5] S. P. Lu and W.G. Ge, "Periodic solutions to a neutral functional differential equation with multiple deviating arguments," Applied Mathematics and Computation, vol. 152, pp. 17-27, 2004.

[6] S. P. Lu and W. G. Ge, "On the existence of periodic solutions for a kind of second order neutral functional differential equation," Acta Mathematica Sinica, vol. 21, no. 2, pp. 381-392, 2005.

[7] S. W. Ma, Z. C. Wang, and J. S. Yu, "Coincidence degree and periodic solutions of Duffing equations," Nonlinear Analysis. Theory, Methods \& Applications, vol. 34, no. 3, pp. 443-460, 1998.

[8] B. W. Liu and L. H. Huang, "Existence and uniqueness of periodic solutions for a kind of first order neutral functional differential equations," Journal of Mathematical Analysis and Applications, vol. 322, no. 1, pp. 121-132, 2006.

[9] J. H. Shen and R. X. Liang, "Periodic solutions for a kind of second order neutral functional differential equations," Applied Mathematics and Computation, vol. 190, no. 2, pp. 1394-1401, 2007.

[10] Y. L. Zhu and S. P. Lu, "Periodic solutions for $p$-Laplacian neutral functional differential equation with deviating arguments," Journal of Mathematical Analysis and Applications, vol. 325, no. 1, pp. 377-385, 2007.
[11] F. Liang, L. X. Guo, and S. P. Lu, "Existence of periodic solutions for a $p$-Laplacian neutral functional differential equation," Nonlinear Analysis. Theory, Methods \& Applications, vol. 71, no. 1-2, pp. 427-436, 2009.

[12] S. P. Lu and W. G. Ge, "Periodic solutions to to a kind of neutral functional differential equations in the critical case," Journal of Mathematical Analysis and Applications, vol. 293, no. 2, pp. 462475, 2004.

[13] S. P. Lu, Z. J. Gui, and W. G. Ge, "Periodic solutions to a second order nonlinear neutral functional differential equation in the critical case," Nonlinear Analysis. Theory, Methods \& Applications, vol. 64, no. 7, pp. 1587-1607, 2006.

[14] S. P. Lu and Z. J. Gui, "On the existence of periodic solutions to Rayleigh differential equation of neutral type in the critical case," Nonlinear Analysis. Theory, Methods \& Applications, vol. 67, no. 4, pp. 1042-1054, 2007.

[15] S. P. Lu, "On the existence of periodic solutions to a $p$-Laplacian neutral differential equation in the critical case," Nonlinear Analysis. Real World Applications, vol. 10, no. 5, pp. 2884-2893, 2009.

[16] E. Müller-Pfeiffer, "Oscillation criteria for selfadjoint fourth order differential equations," Journal of Differential Equations, vol. 46, no. 2, pp. 194-215, 1982.

[17] P. C. Carrião, L. F. O. Faria, and O. H. Miyagaki, "Periodic solutions for extended Fisher-Kolmogorov and Swift-Hohenberg equations by truncature techniques," Nonlinear Analysis. Theory, Methods \& Applications, vol. 67, no. 11, pp. 3076-3083, 2007.

[18] R. P. Agarwal, S. R. Grace, and J. V. Manojlovic, "Oscillation criteria for certain fourth order nonlinear functional differential equations," Mathematical and Computer Modelling, vol. 44, no. 1-2, pp. 163-187, 2006.

[19] F. Li, Y. Li, and Z. Liang, "Existence and multiplicity of solutions to $2 m$ th-order ordinary differential equations," Journal of Mathematical Analysis and Applications, vol. 331, no. 2, pp. 958-977, 2007.

[20] P. Amster and M. C. Mariani, "Oscillating solutions of a nonlinear fourth order ordinary differential equation," Journal of Mathematical Analysis and Applications, vol. 325, no. 2, pp. 1133-1141, 2007.

[21] J. Mawhin and F. Zanolin, "A continuation approach to fourth order superlinear periodic boundary value problems," Topological Methods in Nonlinear Analysis, vol. 2, no. 1, pp. 55-74, 1993.

[22] W. N. Everitt, C. Markett, and L. L. Littlejohn, "Properties of the solutions of the fourth-order Bessel-type differential equation," Journal of Mathematical Analysis and Applications, vol. 359, no. 1, pp. 252-264, 2009.

[23] L. A. Peletier and W. C. Troy, "Spatial patterns described by the extended Fisher-Kolmogorov equation: periodic solutions," SIAM Journal on Mathematical Analysis, vol. 28, no. 6, pp. 13171353, 1997.

[24] S. Jin and S. P. Lu, "Periodic solutions for a fourth-order $p$ Laplacian differential equation with a deviating argument," Nonlinear Analysis. Theory, Methods \& Applications, vol. 69, no. 5-6, pp. 1710-1718, 2008.

[25] S. Tersian and J. Chaparova, "Periodic and homoclinic solutions of extended Fisher-Kolmogorov equations," Journal of Mathematical Analysis and Applications, vol. 260, no. 2, pp. 490-506, 2001.

[26] J. R. Ward, Jr., "Asymptotic conditions for periodic solutions of ordinary differential equations," Proceedings of the American Mathematical Society, vol. 81, no. 3, pp. 415-420, 1981. 
[27] Q. Yao, "Existence, multiplicity and infinite solvability of positive solutions to a nonlinear fourth-order periodic boundary value problem," Nonlinear Analysis. Theory, Methods \& Applications, vol. 63, no. 2, pp. 237-246, 2005.

[28] S. Jin and S. P. Lu, "Periodic solutions for third order $p$ Laplacian equation with a deviating argument," Journal of the Franklin Institute, vol. 346, no. 2, pp. 126-135, 2009.

[29] S. P. Lu and S. Jin, "Existence of periodic solutions for a fourthorder $p$-Laplacian equation with a deviating argument," Journal of Computational and Applied Mathematics, vol. 230, no. 2, pp. 513-520, 2009.

[30] H. Okochi, "On the existence of periodic solutions to nonlinear abstract parabolic equations," Journal of the Mathematical Society of Japan, vol. 40, no. 3, pp. 541-553, 1988.

[31] H. Okochi, "On the existence of anti-periodic solutions to nonlinear parabolic equations in noncylindrical domains," Nonlinear Analysis. Theory, Methods \& Applications, vol. 14, no. 9, pp. 771-783, 1990.

[32] Y. Q. Chen, "On Massera’s theorem for anti-periodic solution," Advances in Mathematical Sciences and Applications, vol. 9, no. 1, pp. 125-128, 1999.

[33] Y. Yin, "Monotone iterative technique and quasilinearization for some anti-periodic problems," Nonlinear World, vol. 3, no. 2, pp. 253-266, 1996.

[34] Y. Yin, "Remarks on first order differential equations with antiperiodic boundary conditions," Nonlinear Times and Digest, vol. 2, no. 1, pp. 83-94, 1995.

[35] A. R. Aftabizadeh, S. Aizicovici, and N. H. Pavel, "On a class of second-order anti-periodic boundary value problems," Journal of Mathematical Analysis and Applications, vol. 171, no. 2, pp. 301-320, 1992.

[36] S. Aizicovici, M. McKibben, and S. Reich, "Anti-periodic solutions to nonmonotone evolution equations with discontinuous nonlinearities," Nonlinear Analysis. Theory, Methods \& Applications, vol. 43, no. 2, pp. 233-251, 2001.

[37] Y. Chen, J. J. Nieto, and D. O’Regan, “Anti-periodic solutions for fully nonlinear first-order differential equations," Mathematical and Computer Modelling, vol. 46, no. 9-10, pp. 1183-1190, 2007.

[38] T. Y. Chen, W. B. Liu, J. J. Zhang, and M. Y. Zhang, "Existence of anti-periodic solutions for Liénard equations," Journal of Mathematical Study, vol. 40, no. 2, pp. 187-195, 2007 (Chinese).

[39] Q. Liu, "Existence of anti-periodic mild solutions for semilinear evolution equations," Journal of Mathematical Analysis and Applications, vol. 377, no. 1, pp. 110-120, 2011.

[40] X. Lv, P. Yan, and D. Liu, "Anti-periodic solutions for a class of nonlinear second-order Rayleigh equations with delays," Communications in Nonlinear Science and Numerical Simulation, vol. 15, no. 11, pp. 3593-3598, 2010.

[41] Y. Li and J. Shu, "Anti-periodic solutions to impulsive shunting inhibitory cellular neural networks with distributed delays on time scales," Communications in Nonlinear Science and Numerical Simulation, vol. 16, no. 8, pp. 3326-3336, 2011.

[42] Y. Chen, J. J. Nieto, and D. O’Regan, “Anti-periodic solutions for evolution equations associated with maximal monotone mappings," Applied Mathematics Letters, vol. 24, no. 3, pp. 302307, 2011.

[43] R. Wu, F. Cong, and Y. Li, "Anti-periodic solutions for second order differential equations," Applied Mathematics Letters, vol. 24, no. 6, pp. 860-863, 2011.

[44] Y. Li and T. Zhang, "Existence and uniqueness of anti-periodic solution for a kind of forced Rayleigh equation with state dependent delay and impulses," Communications in Nonlinear Science and Numerical Simulation, vol. 15, no. 12, pp. 4076-4083, 2010.

[45] F.-J. Delvos and L. Knoche, "Lacunary interpolation by antiperiodic trigonometric polynomials," BIT. Numerical Mathematics, vol. 39, no. 3, pp. 439-450, 1999.

[46] J. Y. Du, H. L. Han, and G. X. Jin, "On trigonometric and paratrigonometric Hermite interpolation," Journal of Approximation Theory, vol. 131, no. 1, pp. 74-99, 2004.

[47] H. L. Chen, "Antiperiodic wavelets," Journal of Computational Mathematics, vol. 14, no. 1, pp. 32-39, 1996.

[48] D. O'Regan, Y. J. Cho, and Y. Q. Chen, Topological Degree Theory and Application, Taylor \& Francis Group, Boca Raton, Fla, USA, 2006. 


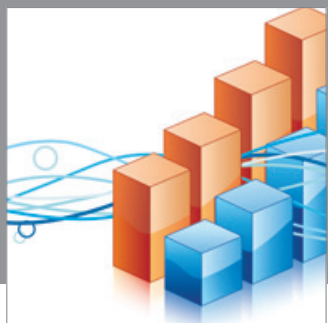

Advances in

Operations Research

mansans

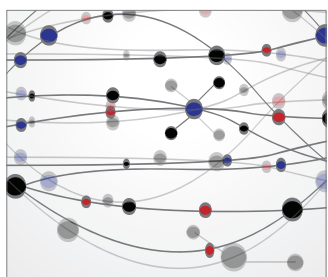

The Scientific World Journal
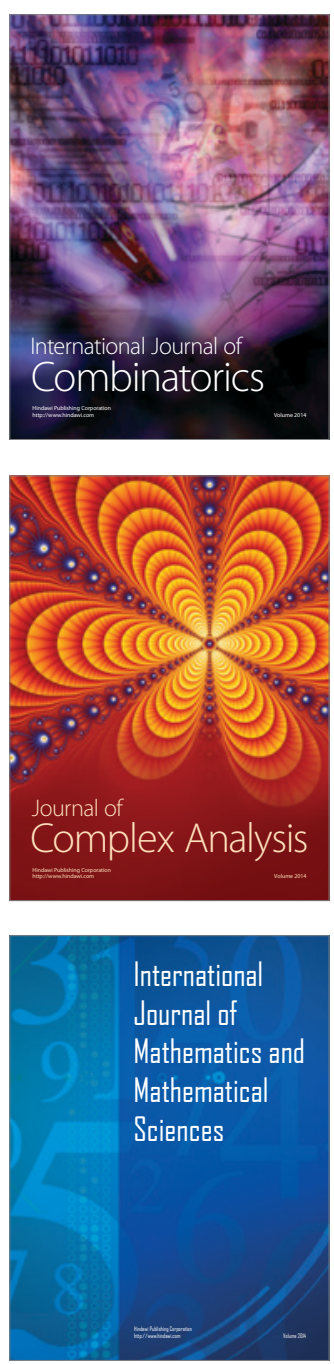
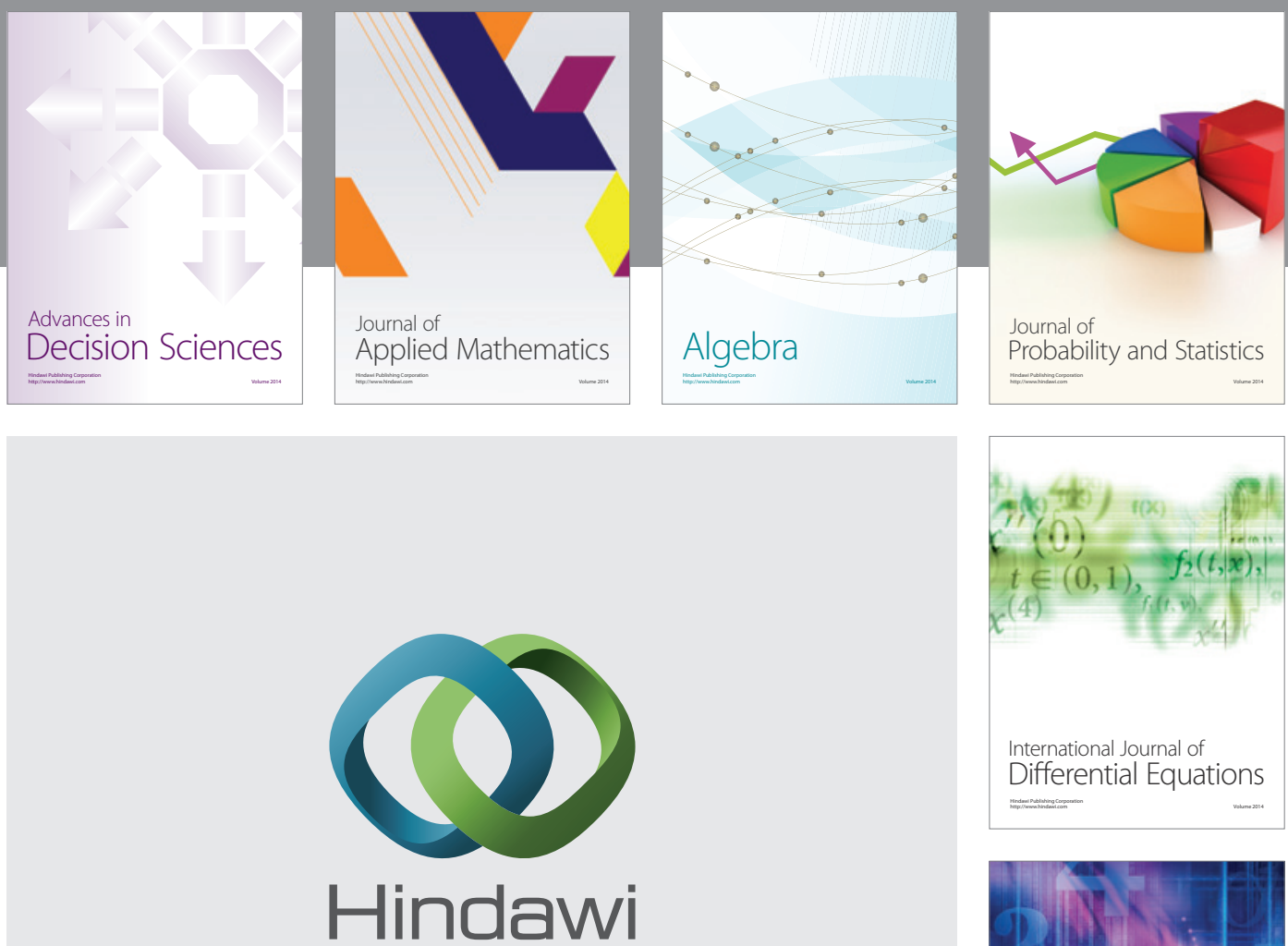

Submit your manuscripts at http://www.hindawi.com
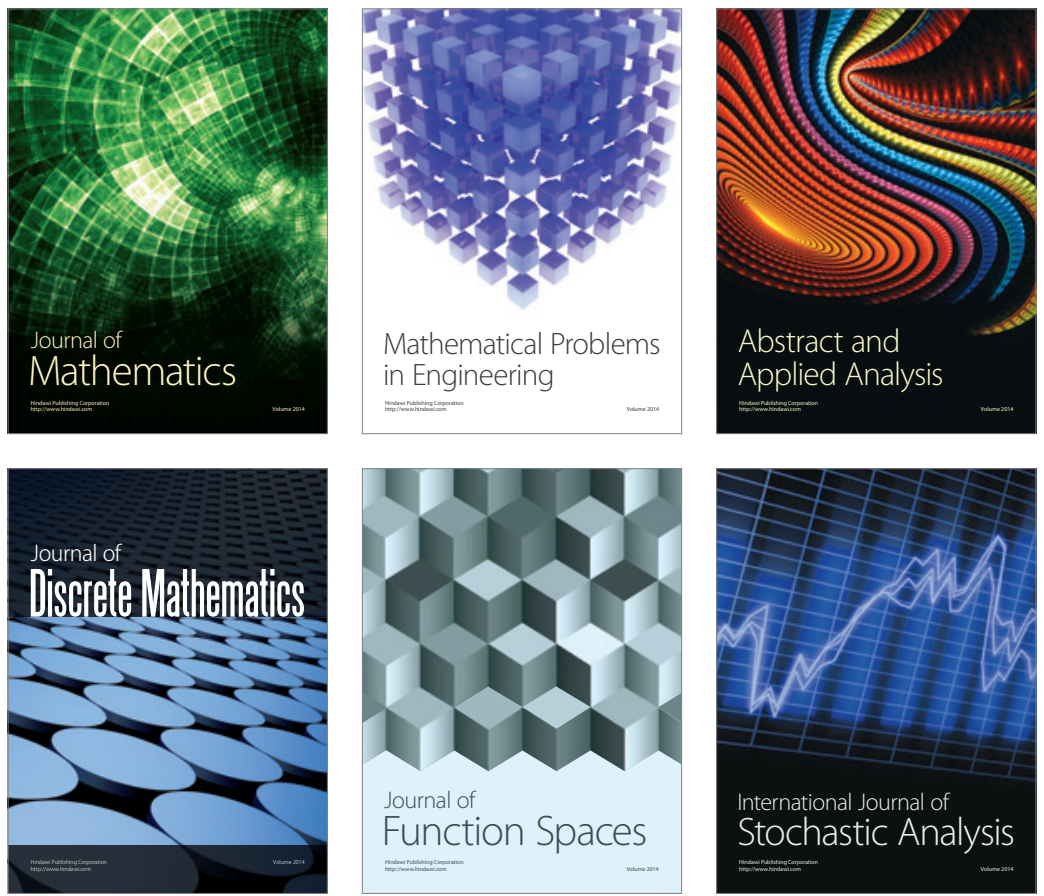

Journal of

Function Spaces

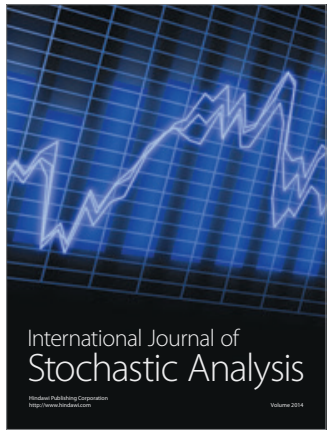

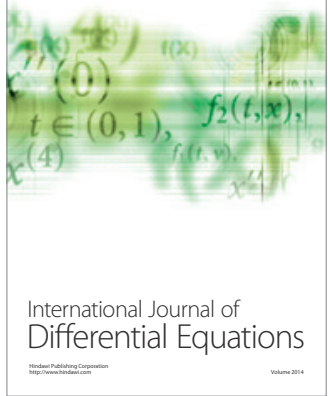
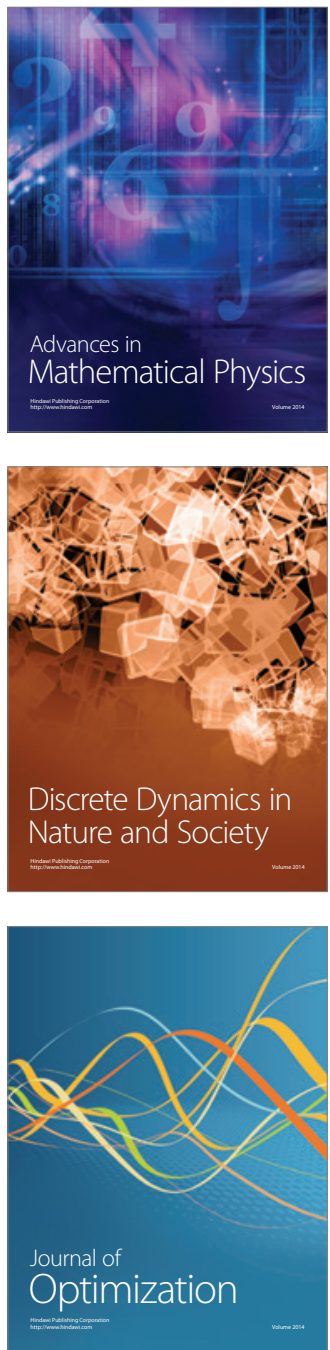